\title{
Further Improvement of Reflection Efficiency of a Magnetic Mirror and Replenishment against Loss of Escaping Deuteron Ions
}

\author{
Mitsuaki Nagata, Keiichi Sawada \\ Soft Creator Company, Kyoto, Japan \\ Email: nagata@heian-kogyo.jp
}

How to cite this paper: Nagata, M. and Sawada, K. (2019) Further Improvement of Reflection Efficiency of a Magnetic Mirror and Replenishment against Loss of Escaping Deuteron Ions. Journal of Modern Physics, 10, 145-156.

https://doi.org/10.4236/jmp.2019.102011

Received: December 6, 2018

Accepted: February 24, 2019

Published: February 27, 2019

Copyright $\odot 2019$ by author(s) and Scientific Research Publishing Inc. This work is licensed under the Creative Commons Attribution International License (CC BY 4.0).

http://creativecommons.org/licenses/by/4.0/

\begin{abstract}
We reported previously the idea to improve reflection-ability of a magnetic mirror by installing a cyclotron resonance space in the front part of the mirror. However, since the previous analysis was insufficient from the examination after that, we complement the following two things in this work: 1) A simpler procedure of design to make a supplemental magnetic mirror with the simplest magnetic configuration, compared with the procedure reported previously. 2) A peculiar characteristic arising only in reflection of a nonrelativistic charged particle (a deuteron ion).
\end{abstract}

\section{Keywords}

Magnetic Mirror, Cyclotron Resonance Heating, Reflection and Replenishment of Deuteron Ions

\section{Introduction}

We found the term seen in the rear of (9) to accelerate only a relativistic charged particle rapidly at a cyclotron resonance point and reported previously [1] applying a work of the term to decreasing the half-vertical angle (called the loss angle) of the loss cone of a magnetic mirror by installing a cyclotron resonance space within. However, based on the examination after that, we would complement two things here. One is about the simplest supplemental magnetic mirror. The procedure of design is mentioned in $\$ 3.1$ together with Figure 1. Another thing is that we missed in Ref. [1] a factor which must be taken into consideration with respect to reflection of a nonrelativistic particle (a deuteron ion). The factor is that an electric field being installed in the front part of a magnetic mir- 
ror does both the work of taking out ions from inside of the loss cone into outside and the reverse work. When we inquired into this problem, we met with a peculiar characteristic with respect to reflection of a deuteron ion. This thing is mentioned in $\$ 3.2$. Finally, we have proposed an idea about a means for replenishing a large quantity of deuteron ions from outside, in order to make the length of a supplemental magnetic mirror as short as possible.

\section{Relativistic Cyclotron Resonance}

We first describe theoretical expressions to be necessary for after analysis. The relativistic equation of motion

$$
\begin{aligned}
& \frac{\partial}{\partial t} \frac{m_{e} \boldsymbol{v}\left(t, t_{0}\right)}{\left[1-v\left(t, t_{0}\right)^{2} / c^{2}\right]^{1 / 2}} \\
& =\frac{m_{e} \partial \boldsymbol{v}\left(t, t_{0}\right) / \partial t}{\left[1-v\left(t, t_{0}\right)^{2} / c^{2}\right]^{1 / 2}}+\frac{\left[m_{e} v\left(t, t_{0}\right) / c^{2}\right] v\left(t, t_{0}\right) \partial v\left(t, t_{0}\right) / \partial t}{\left[1-v\left(t, t_{0}\right)^{2} / c^{2}\right]^{3 / 2}} \\
& =-q \boldsymbol{E}(t)-q \boldsymbol{v}\left(t, t_{0}\right) \times \boldsymbol{B}
\end{aligned}
$$

is changed into (1) below by the help of the following two relationships:

1) The time derivative of energy with respect to a relativistic electron

$$
\begin{aligned}
& \frac{\partial}{\partial t} \frac{m_{e} c^{2}}{\left[1-v\left(t, t_{0}\right)^{2} / c^{2}\right]^{1 / 2}}=\frac{m_{e} v\left(t, t_{0}\right) \partial v\left(t, t_{0}\right) / \partial t}{\left[1-v\left(t, t_{0}\right)^{2} / c^{2}\right]^{3 / 2}} \\
& =-q \boldsymbol{E}(t) \cdot \boldsymbol{v}\left(t, t_{0}\right)
\end{aligned}
$$

2) The integration of the above equation from $t_{0}$ to $t$

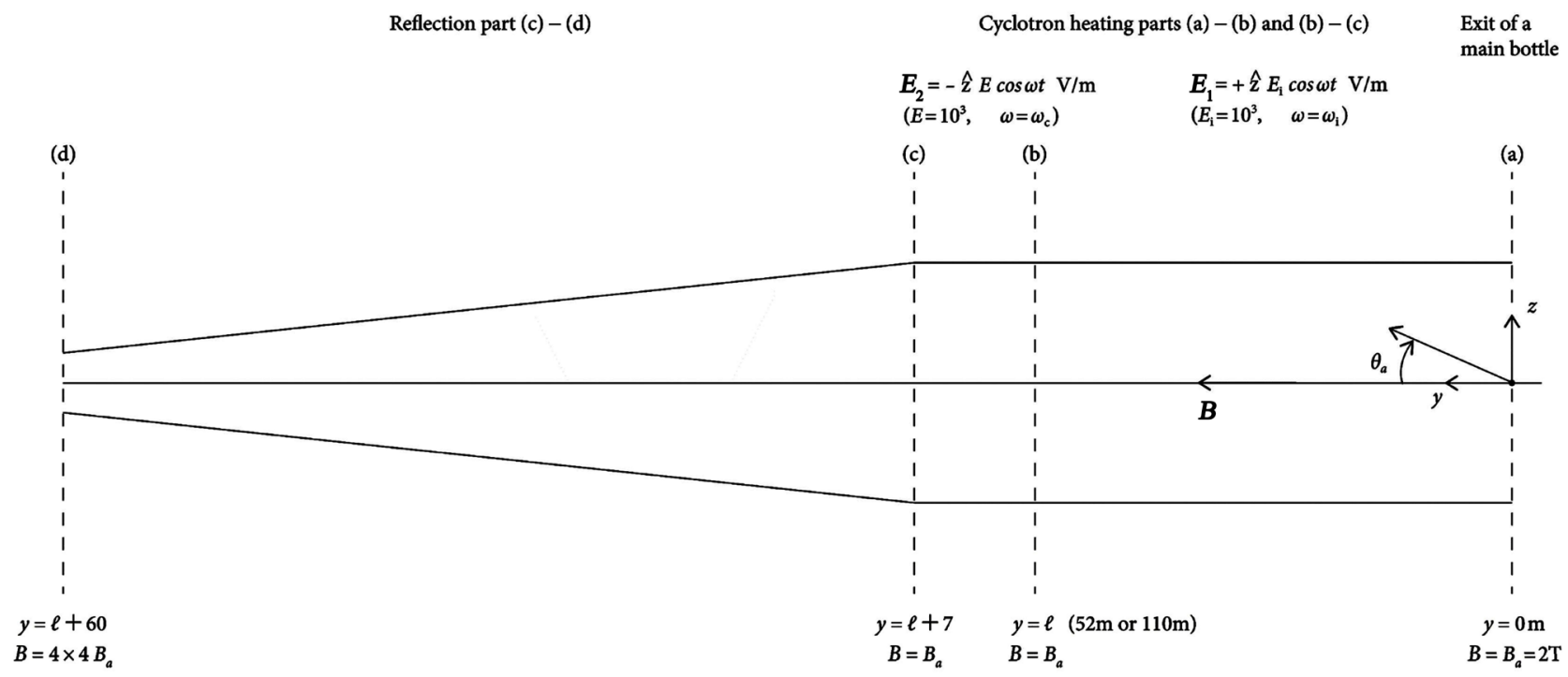

Figure 1. A supplemental magnetic mirror (a)-(d) for reclaiming charged particles to escape from the exit (plane (a)) of the main bottle. The half-vertical angle of the loss cone of mirror (c)-(d) is $14.5^{\circ}$. It is tried to decrease the loss angle of mirror (a)-(d) from $14.5^{\circ}$ to about $5^{\circ}$ with the help of the electric fields $E_{1}$ and $E_{2} . E_{1}$ is the electric field to accelerate deuteron ions, $E_{2}$ is the electric field to accelerate electrons, $\omega_{i}$ is an ion cyclotron frequency and $\omega_{c}$ is an electron cyclotron frequency. 


$$
\begin{gathered}
\frac{m_{e} c^{2}}{\left[1-v\left(t, t_{0}\right)^{2} / c^{2}\right]^{1 / 2}}-\frac{m_{e} c^{2}}{\left[1-\boldsymbol{v}_{0}^{2} / c^{2}\right]^{1 / 2}}=\int_{t_{0}}^{t}-q \boldsymbol{E}(t) \cdot \boldsymbol{v}\left(t, t_{0}\right) \mathrm{d} t \\
\left\{\frac{m_{e}}{\left[1-\boldsymbol{v}_{0}^{2} / c^{2}\right]^{1 / 2}}+\int_{t_{0}}^{t} \frac{-q \boldsymbol{E}(t) \cdot \boldsymbol{v}\left(t, t_{0}\right)}{c^{2}} \mathrm{~d} t\right\} \frac{\partial \boldsymbol{v}\left(t, t_{0}\right)}{\partial t}+\boldsymbol{v}\left(t, t_{0}\right)\left(\frac{-q \boldsymbol{E}(t) \cdot \boldsymbol{v}\left(t, t_{0}\right)}{c^{2}}\right) \\
=-q \boldsymbol{E}(t)-q \boldsymbol{v}\left(t, t_{0}\right) \times \boldsymbol{B}
\end{gathered}
$$

In the above four equations, $m_{e}$ is the rest mass of an electron, $-q$ is the electron charge, $c$ is the speed of light, $E(t)$ is an electric field, $B$ is a magnetic field, $v\left(t, t_{0}\right) \quad\left(=\hat{x} v_{x}\left(t, t_{0}\right)+\hat{y} v_{y}\left(t, t_{0}\right)+\hat{z} v_{z}\left(t, t_{0}\right)\right)$ is the velocity of an electron at time $t$ after start with an initial velocity $v_{0}\left(=\hat{x} v_{0 x}+\hat{y} v_{0 y}+\hat{z} v_{0 z}\right)$ at time $t_{0}$, and $v\left(t, t_{0}\right)=\left|\boldsymbol{v}\left(t, t_{0}\right)\right|$.

Since a solution of $(1), v\left(t, t_{0}\right)$, is to be given by a form of

$$
\boldsymbol{v}\left(t, t_{0}\right)=\boldsymbol{v}_{t}+\boldsymbol{o}\left\{\begin{array}{l}
\boldsymbol{v}_{t}=\boldsymbol{v}\left(t, t_{0}\right)_{(E(t)=0)}, \\
\boldsymbol{o} \text { is a quantity which consists of components of } \boldsymbol{E}(t),
\end{array}\right.
$$

we neglect terms including products and squares with respect to components of $\boldsymbol{E}(t)$ in (1) and linearize (1) as

$$
\begin{aligned}
& \frac{m_{e}}{\left(1-\frac{\boldsymbol{v}_{0}^{2}}{c^{2}}\right)^{\frac{1}{2}}} \frac{\partial \boldsymbol{v}\left(t, t_{0}\right)}{\partial t}+\left(\int_{t_{0}}^{t} \frac{-q \boldsymbol{E}(t) \cdot \boldsymbol{v}_{t}}{c^{2}} \mathrm{~d} t\right) \frac{\partial \boldsymbol{v}_{t}}{\partial t}+\boldsymbol{v}_{t}\left(\frac{-q \boldsymbol{E}(t) \cdot \boldsymbol{v}_{t}}{c^{2}}\right) \\
& =-q \boldsymbol{E}(t)-q \boldsymbol{v}\left(t, t_{0}\right) \times \boldsymbol{B}
\end{aligned}
$$

First, we note the following problem which arises due to the linearization. It is that, in Figure 2 (shown after) for $\hat{z} \cdot v\left(t, t_{0}\right)$ which is obtain from (2), maximum of $\hat{z} \cdot \boldsymbol{v}\left(t, t_{0}\right)$ goes over the speed $c$ of light in long flight time. The magnitude of $v\left(t, t_{0}\right)$ is estimated to be a function increasing together with time $t$ under the resonance condition (an electric field frequency $=\mathrm{a}$ cyclotron frequency), and a mass increment per unit time is given by $-q \boldsymbol{E}(t) \cdot \boldsymbol{v}\left(t, t_{0}\right) / c^{2}$. Accordingly, we consider that the primary cause giving rise to this problem is underestimation in the linearization for the total mass

$\left\{\frac{m_{e}}{\left[1-\boldsymbol{v}_{0}^{2} / c^{2}\right]^{1 / 2}}+\int_{t_{0}}^{t} \frac{-q \boldsymbol{E}(t) \cdot \boldsymbol{v}\left(t, t_{0}\right)}{c^{2}} \mathrm{~d} t\right\}$ at time $t$. A more rapid increase of the total mass ought to reduce variation of $\left|v\left(t, t_{0}\right)\right|$ to zero before $\left|v\left(t, t_{0}\right)\right|$ arrives at the speed $c$ of light.

We solve (2) for $v_{z}\left(t, t_{0}\right)$ and $v_{y}\left(t, t_{0}\right)$ under the following external force fields:

$$
\left\{\begin{array}{l}
\boldsymbol{E}(t)=-\hat{z} E \cos \omega t, \\
\boldsymbol{B}=\hat{y} B,
\end{array}\right.
$$

and under the following initial conditions at $t=t_{0}$ which are given by 
(a) $t_{0}=0$,

$e_{z}$ starting with $\theta_{b}=5^{\circ}$ at $t=0$

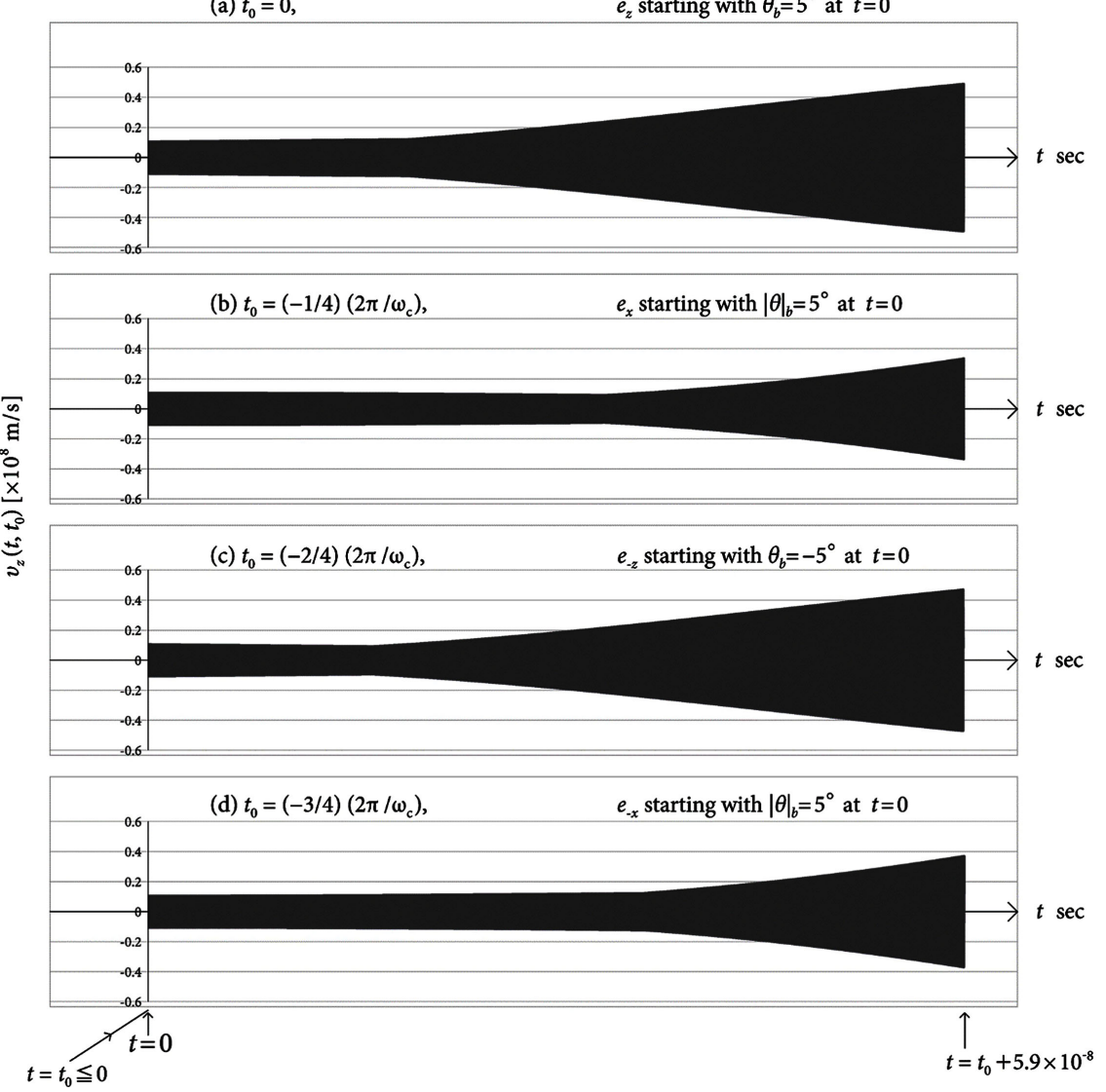

Figure 2. Dependence of $v_{z}\left(t, t_{0}\right)$ on $t_{0}$, based on Equation (6). The numerical conditions are: an initial velocity $\hat{z} v_{0 z}=\hat{z} \bar{v} \sin 5^{\circ}=\hat{z} 0.105 \times 10^{8} \mathrm{~m} / \mathrm{s}$ in plane (b) at time $t_{0}$, $\boldsymbol{E}(t)=-\hat{z} 10^{3} \cos \omega t \mathrm{~V} / \mathrm{m}, \boldsymbol{B}=2 \mathrm{~T}, \omega_{c} / \omega=1.0001$, the time variable

$t=t_{0} \sim t_{0}+7.0 /\left(\bar{v} \cos 5^{\circ}\right)=t_{0} \sim t_{0}+5.9 \times 10^{-8} \mathrm{sec}$. Note that the initial velocity at time $t_{0}$ is $+\hat{z} 0.105 \times 10^{8} \mathrm{~m} / \mathrm{s}$ all in the four curves, but curves (a), (b), (c), (d) can be regarded to show velocity-variations for $e_{z}, e_{x}, e_{-z}, e_{-x}$ starting with each different initial velocity from plane (b) at $t=0\left(\neq t_{0}\right.$, expect for curve (a)).

$$
\left\{\begin{array}{c}
\boldsymbol{v}\left(t, t_{0}\right)_{\left(t=t_{0}\right)}=\hat{z} v_{0 z}+\hat{y} v_{0 y}, \\
\left(\frac{\partial v_{z}\left(t, t_{0}\right)}{\partial t}\right)_{\left(t=t_{0}\right)}=\frac{q E}{m} \cos \omega t_{0}-\frac{q E}{m c^{2}} v_{0 z}^{2} \cos \omega t_{0}, \\
\left(\text { where } m=m_{e}\left(1-v_{0}^{2} / c^{2}\right)^{-1 / 2}\right) .
\end{array}\right.
$$

The results are given in (5) and (6) below,

$$
v_{y}\left(t, t_{0}\right)=v_{0 y}+v_{0 y} v_{0 z} \frac{q E}{m c^{\prime \prime}}\left\{\frac{\sin \left[\left(\omega_{c}+\omega\right)\left(t-t_{0}\right)\right]}{2\left(\omega_{c}+\omega\right)}+\frac{\sin \left[\left(\omega_{c}-\omega\right)\left(t-t_{0}\right)\right]}{2\left(\omega_{c}-\omega\right)}\right\} \text {. }
$$

Here, $c^{\prime \prime}=c^{2}$ (The speed $c$ of light appears always in the form of square in after analysis. Then, we have introduced the symbol $c^{\prime \prime}$ for $c^{2}$, because we would like to use $c$ as the symbol for $\cos \omega t$ ), and $\omega_{c}=q B / m$. Since the second term 
of (5) is extremely small compared with the first term $v_{0 y}$ in after analysis under the conditions of $\omega \simeq \omega_{c}$ and $\omega_{c}\left(t-t_{0}\right) \gg 1, v_{y}\left(t, t_{0}\right)$ is regarded to be constant $v_{0 y}$ in this work.

$$
v_{z}\left(t, t_{0}\right)=a_{1} s+\left(a_{2} s\right) S^{2}+\left(a_{3} s\right) C^{2}+\left(a_{4} c\right) S C+a_{5} t S+C_{1} C+C_{2} S,
$$

(We have used $v_{t}\left(=\hat{x} v_{0 z} \sin \omega_{c}\left(t-t_{0}\right)+\hat{y} v_{0 y}+\hat{z} v_{0 z} \cos \omega_{c}\left(t-t_{0}\right)\right)$ ).

Here,

$$
\begin{gathered}
C=\cos \omega_{c}\left(t-t_{0}\right), S=\sin \omega_{c}\left(t-t_{0}\right), c=\cos \omega t, s=\sin \omega t, \\
a_{1}=\frac{q E}{m} \frac{-\omega}{\omega_{c}^{2}-\omega^{2}}, \\
a_{2}=\frac{q E}{m c^{\prime \prime}} \frac{1}{\left(\omega_{c}^{2}-\omega^{2}\right)^{2}}\left[-2 \omega_{c}^{2} \omega v_{0 z}^{2}\right], \\
a_{3}=\frac{q E}{m c^{\prime \prime}} \frac{1}{\left(\omega_{c}^{2}-\omega^{2}\right)^{2}}\left[\left(\omega_{c}^{2} \omega-\omega^{3}\right) v_{0 z}^{2}\right], \\
a_{4}=\frac{q E}{m c^{\prime \prime}} \frac{2 \omega_{c}^{3}}{\left(\omega_{c}^{2}-\omega^{2}\right)^{2}}\left(-v_{0 z}^{2}\right), \\
a_{5}=\frac{q E}{m c^{\prime \prime}} \frac{\omega_{c}}{\omega_{c}^{2}-\omega^{2}}\left(v_{0 z}^{2} \omega \sin \omega t_{0}\right), \\
C_{1}=v_{0 z}-a_{1} \sin \omega t_{0}-a_{3} \sin \omega t_{0}, \\
C_{2}=\frac{q E}{m \omega_{c}} \cos \omega t_{0}-\frac{q E}{m c^{\prime \prime}} \frac{1}{\omega_{c}} v_{0 z}^{2} \cos \omega t_{0}-a_{1} \frac{\omega}{\omega_{c}} \cos \omega t_{0} \\
-a_{3} \frac{\omega}{\omega_{c}} \cos \omega t_{0}-a_{4} \cos \omega t_{0}-a_{5} t_{0} .
\end{gathered}
$$

Next let us obtain velocities perpendicular to the magnetic field at the resonance point $\omega \rightarrow \omega_{c}$, in the simple case where $t_{0}=0$ :

$$
\begin{gathered}
v_{z r} \equiv \lim _{\omega \rightarrow \omega_{c}} v_{z}\left(t, t_{0}\right)_{\left(t_{0}=0\right)}=\lim _{\omega \rightarrow \omega_{c}}(6)_{\left(t_{0}=0\right)} \\
=v_{0 z} C_{0}+\frac{q E}{m}\left(1-\frac{v_{0 z}^{2}}{c^{\prime \prime}}\right) \frac{1}{\omega_{c}} S_{0}+\frac{q E}{m}\left(\frac{1}{-2 \omega_{c}}\left(S_{0}-\omega_{c} t C_{0}\right)\right) \\
+\frac{q E}{m} \frac{v_{0 z}^{2}}{c^{\prime \prime}}\left(\frac{1}{8 \omega_{c}^{2}}\left(2 \omega_{c}^{3} t^{2} S_{0}-4 \omega_{c}^{2} t C_{0}+4 \omega_{c} S_{0}+6 \omega_{c} S_{0}^{3}\right)\right), \\
v_{x r} \equiv \lim _{\omega \rightarrow \omega_{c}} v_{x}\left(t, t_{0}\right)_{\left(t_{0}=0\right)} \\
=v_{0 z} S_{0}+\frac{1}{2} \frac{q E}{m} t S_{0}+\frac{q E}{m} \frac{v_{0 z}^{2}}{c^{\prime \prime}}\left(\frac{1}{8 \omega_{c}^{2}}\left(-4 \omega_{c}^{2} t S_{0}-2 \omega_{c}^{3} t^{2} C_{0}-6 \omega_{c} S_{0}^{2} C_{0}\right)\right), \\
v_{r} \equiv\left(v_{z r}^{2}+v_{x r}^{2}\right)^{\frac{1}{2}} \simeq\left\{\left[v_{0 z}+\frac{q E t}{2 m}\left(1-\frac{v_{0 z}^{2}}{c^{\prime \prime}}\right)\right]^{2}+\left[\frac{q E t}{m} \cdot \frac{v_{0 z}^{2}}{c^{\prime \prime}} \cdot \frac{\omega_{c} t}{4}\right]^{2}\right\}^{1 / 2}, \quad\left(\omega_{c} t \gg 1\right) .
\end{gathered}
$$

Here, $C_{0}=C_{\left(t_{0}=0\right)}, S_{0}=S_{\left(t_{0}=0\right)}$, and $v_{x}\left(t, t_{0}\right)_{\left(t_{0}=0\right)}$ is given in Equation (15) of Ref. [1]. It should be noted that the second term within the root in (9) works 
greatly only for a relativistic charged particle but the relativistic work of the second term will reduce more and more than the estimation in (9) as time $t$ passes, because of the underestimation in (2) for the mass increment $-q \boldsymbol{E}(t) \cdot \boldsymbol{v}\left(t, t_{0}\right) / c^{\prime \prime}$ per unit time. Also, it must be noted that there is some difference between $v_{r}$ of (9) and an amplitude of a curve of $\hat{z} \cdot \boldsymbol{v}\left(t, t_{0}\right)$ seen in Figure 2 (discussed in \$3.1). However, we try designing a magnetic mirror for electrons based on a time-variation of an amplitude of $\hat{z} \cdot v\left(t, t_{0}\right)$.

\section{Design of a Supplemental Magnetic Mirror}

In order to return back deuteron ions (called $\mathrm{D}^{+}$ions) and electrons escaping from a main bottle as many as possible, we consider installing (at the exit of the main bottle) a supplemental magnetic mirror which has a cyclotron resonance space within, as shown in Figure 1. We intend to reduce a loss angle of the supplemental mirror by increasing magnitudes of velocities perpendicular to a magnetic field $\boldsymbol{B}$ of the escaping particles within the cyclotron resonance space [2] [3] [4] [5]. The supplemental mirror is divided to three spaces by plane ( $j)(j=$ a, b, c, d). We define the $x, y, z$ coordinate-system, as shown in plane (a). Also, we assume the $y$-coordinate and a strength $B$ of a magnetic field $B$ in each plane as shown in the figure. The magnetic field is regarded to be only in the $+y$-direction. Electric fields $\boldsymbol{E}_{1}$ and $\boldsymbol{E}_{2}$ are supplied within spaces (a) - (b) and (b) - (c), respectively. For an incident angle when a charged particle crosses plane (j), we denote an angle from $+y$-axis in the $y$ - $z$ plane by $\theta_{j} \quad\left(-90^{\circ}<\theta_{j}<90^{\circ}\right)$ and a mere inclination from $+y$-axis by $|\theta|_{j} \quad\left(0 \leq|\theta|_{j}<90^{\circ}\right)$. A plasma temperature for fusion reaction to continue is assumed to be $4 \times 10^{8} \mathrm{~K}$. Then, each mean thermal velocity for electrons and $\mathrm{D}^{+}$ions is about $1.2 \times 10^{8} \mathrm{~m} / \mathrm{s}(=\bar{v})$ and $2 \times 10^{6} \mathrm{~m} / \mathrm{s}$ $\left(=\bar{v}_{i}\right)$, respectively. To simplify after discussion, we assume that every electron and every $\mathrm{D}^{+}$ion are flying about within the main bottle, with each mean thermal velocity $\bar{v}$ and $\bar{v}_{i}$. Also, we disregard interactions between charged particles through Coulomb force within the supplemental mirror. Also in Figure 1, an electron or a $\mathrm{D}^{+}$ion is regarded to actually interact only with the electric field having $\omega_{c}$ or $\omega_{i}$, respectively.

\subsection{Reflection of Electrons}

First we show in Figure 2 the $z$-component of velocity, $v_{z}\left(t, t_{0}\right)$ given in (6), in the time range of $\left(t_{0} \leq t \leq t_{0}+5.9 \times 10^{-8} \mathrm{sec}\right)$ for an electron which starts from plane (b) with an initial velocity $\hat{z} \bar{v} \sin \theta_{b}+\hat{y} \bar{v} \cos \theta_{b}$

$\left(\bar{v}=1.2 \times 10^{8} \mathrm{~m} / \mathrm{s}, \theta_{b}=+5^{\circ}\right)$ at time $t_{0}$. The numerical conditions are shown in the figure. Curves (a), (b), (c), (d) are for four cases of $t_{0}$. Here, we note the following thing. In the short time range of $t_{0} \sim 0$ (note that $t_{0} \leq 0$ ), both the velocity-magnitude and the gyration frequency of the electron hardly vary compared with each initial value at $t=t_{0}$. Therefore, since $\omega_{c} \simeq \omega$, curves (a), (b), (c), (d) can be regarded to show time-variations of the $z$-components of velocities perpendicular to $\boldsymbol{B}$ for four electrons (called $e_{z}, e_{x}, e_{-z}, e_{-x}$ ), starting from plane (b) with initial velocities shown below at time $t=0\left(\neq t_{0}\right.$, except for curve 
(a)):

1) $\hat{z} \bar{v} \sin \theta_{b}+\hat{y} \bar{v} \cos \theta_{b}\left(\theta_{b}>0\right)$ for $e_{z}$,

2) $\hat{x} \bar{v} \sin |\theta|_{b}+\hat{y} \bar{v} \cos |\theta|_{b}\left(|\theta|_{b}=\left|\theta_{b}\right|\right)$ for $e_{x}$,

3) $\hat{z} \bar{v} \sin \theta_{b}+\hat{y} \bar{v} \cos \theta_{b}\left(\theta_{b}<0\right)$ for $e_{-z},\left(\theta_{b}= \pm 5^{\circ}\right.$ in Figure 2)

4) $-\hat{x} \bar{v} \sin |\theta|_{b}+\hat{y} \bar{v} \cos |\theta|_{b}\left(|\theta|_{b}=\left|\theta_{b}\right|\right)$ for $e_{-x}$,

(Note that the above four velocities are initial velocities at $t=0$ all).

In the characteristics of curve (a) for $e_{z}$ and curve (c) for $e_{-z}$, the difference is hardly seen, which is due to that the second term within the root in Equation (9) is much more predominant than the first term within the root in magnitude. We consider making these four electrons reflect by mirror (c) - (d) all. For this purpose, the four electrons must satisfy the following reflection condition:

$$
\frac{v_{r(c)}}{\left(v_{r(c)}^{2}+\bar{v}^{2} \cos ^{2} \theta_{c}\right)^{1 / 2}}>\left(\frac{B_{a}}{4 \times 4 B_{a}}\right)^{1 / 2} \text { or } v_{r(c)}>0.26 \bar{v} \cos \theta_{c} \simeq 0.26 \bar{v} \cos \theta_{b}
$$

(The loss angle of mirror (c) - (d) is $14.5^{\circ}$ ).

Here, $4 \times 4 B_{a}$ is the magnetic field in plane (d) and $v_{r(c)}$ is a minimum in velocity-magnitudes perpendicular to $\mathbf{B}$ of the four electrons when those cross plane (c). Accordingly, the electric field $\boldsymbol{E}_{2}\left(=-\hat{z} 10^{3} \cos \omega t, \omega_{c} / \omega=1.0001\right)$ in Figure 1 must increase the value of $v_{r(c)}$ from

$\bar{v}\left|\sin \theta_{b}\right|\left(=\bar{v}\left|\sin \pm 5^{\circ}\right|=0.105 \times 10^{8} \mathrm{~m} / \mathrm{s}\right)$ at time $t=0$ to $0.26 \bar{v} \cos \theta_{b} \quad(=0.31 \times$ $\left.10^{8} \mathrm{~m} / \mathrm{s}\right)$. We regard the amplitudes of $v_{z}\left(t, t_{0}\right)$ in curves (a), (b), (c), (d) to show time-variations of velocity-magnitudes perpendicular to $\boldsymbol{B}$ of $e_{z}, e_{x}, e_{-z}, e_{-x}$, respectively. Then, the velocity-magnitudes in curves (a), (b), (c), (d) become larger than $0.26 \cos \theta_{b}=0.31 \times 10^{8} \mathrm{~m} / \mathrm{s}$ all when $t \simeq t_{0}+5.9 \times 10^{-8} \mathrm{sec}$ (A necessary length between planes (b) and (c) is about $7 \mathrm{~m})$. If the four electrons are reflected by mirror (c) - (d), we estimate that the most of electrons with $|\theta|_{b}=5^{\circ} \sim 90^{\circ}$ will return to the main bottle.

Here, we would note the following thing. We tried obtaining from (9) values corresponding to the maxima of $v_{z}\left(t, t_{0}\right)$ near $t=t_{0}+5.9 \times 10^{-8} \simeq 5.9 \times 10^{-8} \mathrm{sec}$ (because of $-\pi / \omega_{c} \simeq-10^{-11} \mathrm{sec}$ ) which are seen in curves (a) and (c) in Figure 2 which is drawn based on (6).

The maxima of $v_{z}\left(t, t_{0}\right) \simeq\left\{\begin{array}{l}0.51 \times 10^{8} \mathrm{~m} / \mathrm{s} \text { in curve }(a), \\ 0.49 \times 10^{8} \mathrm{~m} / \mathrm{s} \text { in curve }(c) .\end{array}\right.$

Substituting into (9)

$$
\left\{\begin{array}{l}
c^{\prime \prime}=\left(3 \times 10^{8} \mathrm{~m} / \mathrm{s}\right)^{2}, E=10^{3} \mathrm{~V} / \mathrm{m}, B=2 \mathrm{~T}, \\
\bar{v}=1.2 \times 10^{8} \mathrm{~m} / \mathrm{s}, \\
v_{0 z}=\bar{v} \sin \theta_{b}=\left\{\begin{array}{l}
0.105 \times 10^{8} \mathrm{~m} / \mathrm{s} \quad \theta_{b}=+5^{\circ} \text { in curves }(a), \\
-0.105 \times 10^{8} \mathrm{~m} / \mathrm{s} \quad \theta_{b}=-5^{\circ} \text { in curves }(c), \\
v_{0 y}=\bar{v} \cos \theta_{b}=1.192 \times 10^{8} \mathrm{~m} / \mathrm{s}, \\
q / m=1.76 \times 10^{11} \times\left(1-\left(v_{0 z}^{2}+v_{0 y}^{2}\right) / c^{\prime \prime}\right)^{1 / 2}=1.61 \times 10^{11} \mathrm{C} / \mathrm{kg}, \\
\omega_{c}=q B / m=3.22 \times 10^{11} \sec ^{-1}, t=5.9 \times 10^{-8} \mathrm{sec},
\end{array}\right.
\end{array}\right.
$$


we obtain

$$
v_{r}= \begin{cases}0.57 \times 10^{8} \mathrm{~m} / \mathrm{s} \text { for curve }(\mathrm{a}), \\ 0.55 \times 10^{8} \mathrm{~m} / \mathrm{s} \text { for curve }(\mathrm{c}) .\end{cases}
$$

There are some differences between the maxima in curves (a), (c) and the above values of $v_{r}$. Then, considering that the differences have come from some approximation, we re-examined the calculation process from (15) of Ref. [1] and (6) to (7) and (8). However, there were no mathematical approximations. On the other hand, in the process from (7) and (8) to (9), there were two approximations below:

$$
\left.\begin{array}{l}
\frac{1}{2} \frac{q E}{m \omega_{c}}\left(1-\frac{v_{0 z}^{2}}{c^{\prime \prime}}\right) S_{0} \rightarrow 0, \\
\frac{1}{4} \frac{q E}{m} \frac{v_{0 z}^{2}}{c^{\prime \prime}}\left(\frac{3 S_{0}^{2}}{\omega_{c}}\right) C_{0} \rightarrow 0,
\end{array}\right\}\left(\omega_{c} t \rightarrow \infty\right) .
$$

However, the above approximations are right when $\omega_{c} t \simeq 2 \times 10^{4}$. So, we consider that the cause of " $v_{r}>$ the maximum of $v_{z}\left(t, t_{0}\right)$ " is in that, of $\left(v_{z r}^{2}+v_{x r}^{2}\right)^{1 / 2}$ in (9), $v_{x r}$ is not zero when the magnitude of $v_{z r}$ becomes maximum.

\subsection{Reflection of $\mathrm{D}^{+}$Ions}

We aim a $\mathrm{D}^{+}$ion which starts from plane (a) in Figure 1 with an initial velocity $\hat{z} v_{i o z}+\hat{y} v_{i o y}=\hat{z} \bar{v}_{i} \sin \theta_{a}+\hat{y} \bar{v}_{i} \cos \theta_{a}$ at time $t_{0}$ and goes to plane (b). An expression (called Equation (11)) for a $\mathrm{D}^{+}$ion corresponding to (6) is obtained by the following change for the symbols of an electron:

$$
\left\{\begin{array}{l}
-q \rightarrow q,-E \rightarrow E_{i}, v_{0 z} \rightarrow v_{i 0 z}\left(=\bar{v}_{i} \sin \theta_{a}\right), \\
m_{e} \rightarrow m_{i}\left(=3680 m_{e}\right), m \rightarrow m_{i i}\left(=m_{i} /\left(1-\left(v_{i 0 z}^{2}+v_{i 0 y}^{2}\right) / c^{\prime \prime}\right)^{1 / 2}\right), \\
\omega_{c} \rightarrow \omega_{i}\left(=q B / m_{i i}\right), v_{z}\left(t, t_{0}\right) \rightarrow v_{i z}\left(t, t_{0}\right) .
\end{array}\right.
$$

We show in Figure 3 dependences of $v_{i z}\left(t, t_{0}\right)$ on four cases of time $t_{0}$, for a $\mathrm{D}^{+}$ion which starts from plane (a) with an initial velocity $\hat{z} \bar{v}_{i} \sin \theta_{a}+\hat{y} \bar{v}_{i} \cos \theta_{a}\left(\theta_{a}=+3^{\circ}\right)$ at time $t_{0}$. The numerical conditions are shown in the figure. Based on the same consideration with in $\$ 3.1$, the amplitudes in curves (a), (b), (c), (d) in Figure 3 are regarded to show time-variations of velocity-magnitudes perpendicular to $\boldsymbol{B}$ of four $\mathrm{D}^{+}$ions (called $\mathrm{D}_{z}^{+}, \mathrm{D}_{x}^{+}, \mathrm{D}_{-z}^{+}, \mathrm{D}_{-x}^{+}$) starting from plane (a) with initial velocities shown below at time $t=0\left(\neq t_{0}\right.$, except for curve (a)):

1) $\hat{z} \bar{v}_{i} \sin \theta_{a}+\hat{y} \bar{v}_{i} \cos \theta_{a}\left(\theta_{a}>0\right)$ for $\mathrm{D}_{z}^{+}$,

2) $\hat{x} \bar{v}_{i} \sin |\theta|_{a}+\hat{y} \bar{v}_{i} \cos |\theta|_{a}\left(|\theta|_{a}=\left|\theta_{a}\right|\right)$ for $\mathrm{D}_{x}^{+},\left(\theta_{a}= \pm 3^{\circ}\right.$ in Figure 3$)$,

3) $\hat{z} \bar{v}_{i} \sin \theta_{a}+\hat{y} \bar{v}_{i} \cos \theta_{a}\left(\theta_{a}<0\right)$ for $\mathrm{D}_{-z}^{+}$,

4) $-\hat{x} \bar{v}_{i} \sin |\theta|_{a}+\hat{y} \bar{v}_{i} \cos |\theta|_{a}\left(|\theta|_{a}=\left|\theta_{a}\right|\right)$ for $\mathrm{D}_{-x}^{+}$,

(Note that the above four velocities are initial velocities at $t=0$ all).

The amplitude in curve (c) changes from decrease into increase halfway. This 
variation can be explained based on an expression $\left(v_{r} \rightarrow v_{i r}\right)$ for a $\mathrm{D}^{+}$ion corresponding to (9):

$$
v_{i r} \simeq\left\{\left[v_{i 0 z}+\frac{q E_{i} t}{2 m_{i i}}\left(1-\frac{v_{i 0 z}^{2}}{c^{\prime \prime}}\right)\right]^{2}+\left[\frac{q E_{i} t}{m_{i i}} \frac{v_{i 0 z}^{2}}{c^{\prime \prime}} \frac{\omega_{i} t}{4}\right]^{2}\right\}^{1 / 2}, \quad\left(\omega_{i} t \gg 1\right)
$$

Since $\bar{v}_{i}^{2} / c^{\prime \prime}=4.4 \times 10^{-5},(12)$ is approximated as

$$
v_{i r} \simeq\left|\bar{v}_{i} \sin \theta_{a}+\frac{q E_{i} t}{2 m_{i}}\right|, \quad\left(\omega_{i} t \gg 1\right)
$$

Since $\theta_{a}=-3^{\circ}$ in curve (c) for $\mathrm{D}_{-z}^{+}$in Figure 3, (13) becomes zero when $\left|\bar{v}_{i} \sin \left(-3^{\circ}\right)\right|=q E_{i} t /\left(2 m_{i}\right)$.

Now, we consider again making four $\mathrm{D}^{+}$ions $\left(\mathrm{D}_{z}^{+}, \mathrm{D}_{x}^{+}, \mathrm{D}_{-z}^{+}, \mathrm{D}_{-x}^{+}\right)$reflect by mirror (c) - (d) all. Denoting by $v_{i r(b)}$ a minimum in velocity-magnitudes perpendicular to $\boldsymbol{B}$ of the four $\mathrm{D}^{+}$ions when those cross plane (b), $v_{i r(b)}$ must satisfy

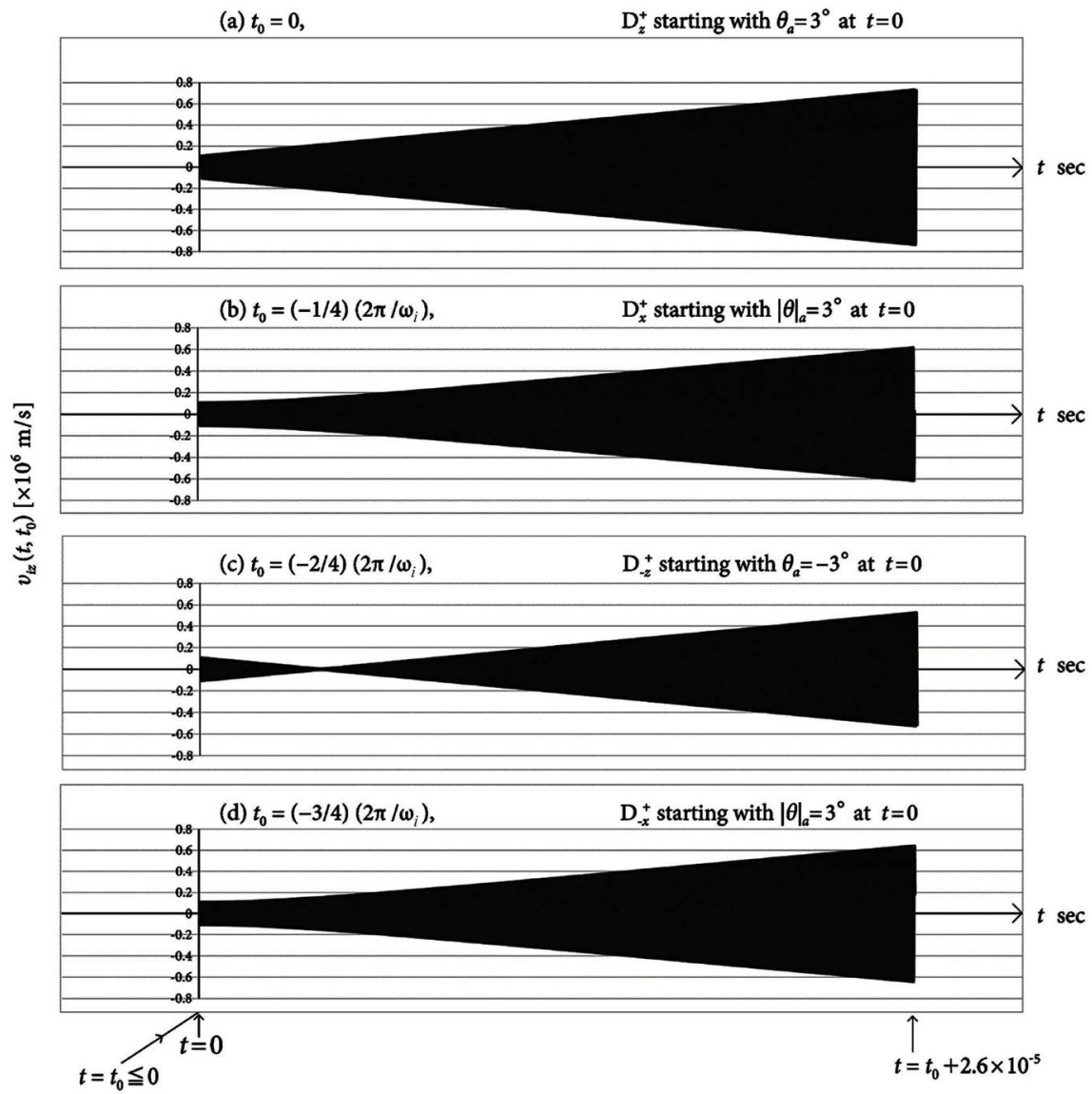

Figure 3. Dependence of $v_{i z}\left(t, t_{0}\right)$ on $t_{0}$, based on Equation (11). The numerical conditions are: An initial velocity $\hat{z} v_{i 0 z}=\hat{z} \bar{v}_{i} \sin 3^{\circ}=\hat{z} 0.105 \times 10^{6} \mathrm{~m} / \mathrm{s}$ in plane (a) at time $t_{0}$, $\boldsymbol{E}_{1}=+\hat{z} 10^{3} \cos \omega t \mathrm{~V} / \mathrm{m}, \boldsymbol{B}=2 \mathrm{~T}, \omega_{i} / \omega=1.0001$, the time variable $t=t_{0} \sim t_{0}+\ell /\left(\bar{v}_{i} \cos 3^{\circ}\right)=t_{0} \sim t_{0}+52 /\left(2 \times 10^{6} \times \cos 3^{\circ}\right)=t_{0} \sim t_{0}+2.6 \times 10^{-5} \mathrm{sec}$. 


$$
v_{i r(b)}>0.26 \bar{v}_{i} \cos \theta_{b} \simeq 0.26 \bar{v}_{i} \cos \theta_{a} \equiv v_{\text {reflect }} .
$$

Accordingly, the electric field $\boldsymbol{E}_{1}\left(=+\hat{z} 10^{3} \cos \omega t, \omega_{i} / \omega=1.0001\right)$ in Figure 1 must increase the value of $v_{i r(b)}$ from $\bar{v}_{i}\left|\sin \theta_{a}\right|\left(=\bar{v}_{i}\left|\sin \left( \pm 3^{\circ}\right)\right|=0.105 \times 10^{6} \mathrm{~m} / \mathrm{s}\right)$ at time $t=0$ to $v_{\text {reflect }}$ of $(14)$ $\left(=0.26 \bar{v}_{i} \cos \left( \pm 3^{\circ}\right)=0.52 \times 10^{6} \mathrm{~m} / \mathrm{s}\right)$. From the characteristics in Figure 3 , the condition mentioned above is satisfied when an acceleration time is about $2.6 \times$ $10^{-5} \mathrm{sec}$ (A necessary length $\ell$ between planes (a) and (b) is about $52 \mathrm{~m}$ ) and mirror (c) - (d) can reflect the four $\mathrm{D}^{+}$ions $\left(\mathrm{D}_{z}^{+}, \mathrm{D}_{x}^{+}, \mathrm{D}_{-z}^{+}, \mathrm{D}_{-x}^{+}\right)$. However, when we had examined about reflection-characteristics of a $\mathrm{D}^{+}$ion (in the range of $-90^{\circ}<\theta_{a} \leq 0^{\circ}$ in the $y-z$ plane) starting from plane (a) at $t=0$, we met with a peculiar dependence on $\theta_{a}$. We missed this factor in Ref. [1]. To mention this thing with respect to a $\mathrm{D}^{+}$ion, we show in Figure 4(a), Figure 4(b) dependences (on $\theta_{a}$ ) of the following four quantities in plane (b):

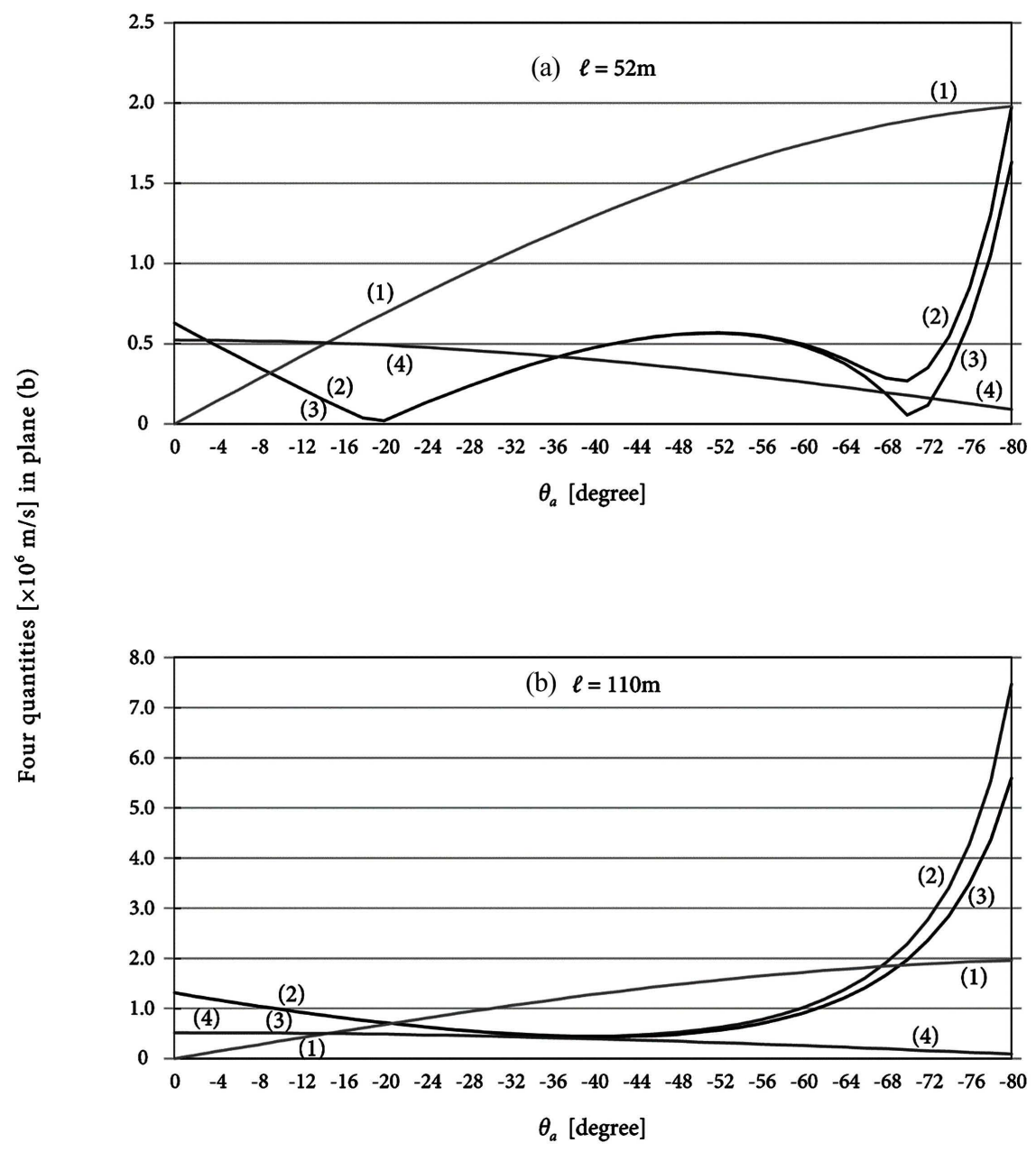

Figure 4. (a) (b) Dependence of four kinds of velocities perpendicular to the magnetic field with respect to reflection of a $\mathrm{D}^{+}$ion on $\theta_{a}\left[0^{\circ} \geq \theta_{a} \geq-80^{\circ}\right]$. (a) is for $\ell=52 \mathrm{~m}$ and (b) is for $\ell=110 \mathrm{~m}$, where $\ell$ is the length between planes (a) and (b) as shown in Figure 1 . (1) $\left|\bar{v}_{i} \sin \theta_{a}\right|$, (2) $v_{i r}$ of Equation (12), (3) $v_{i r}$ of Equation (13), (4) $v_{\text {reflect }}$ of Equation (14). 
1) $\left|\bar{v}_{i} \sin \theta_{a}\right|$, which is the velocity-magnitude perpendicular to $B$ of the initial velocity in plane (a) at $t=0$. This value is constant when $\boldsymbol{E}_{1}=0$.

2) $v_{i r}$ of Equation (12), which is the velocity-magnitude perpendicular to $\boldsymbol{B}$ in plane (b) when $\omega_{i} / \omega=1.0001$.

3) $v_{i r}$ of Equation (13) (= Equation (12) with $c^{\prime \prime} \rightarrow \infty$ ).

4) $v_{\text {reflect }}$ of Equation (14). This is the minimum of $v_{i r}$ in plane (b) which is required for a $\mathrm{D}^{+}$ion to be reflected by mirror (c) - (d).

It was a peculiar variation that, in curves (2) and (3) of Figure 4(a), minima had appeared at two points of $\theta_{a}$.

In Figure 4(a), Figure 4(b), when $\boldsymbol{E}_{1}=0$, from curves (1) and (4), a $\mathrm{D}^{+}$ion in the range of $0^{\circ} \geq \theta_{a}>-14.5^{\circ}$ are not reflected but those in the range of $-14.5^{\circ} \gtrsim \theta_{a} \geq-80^{\circ}$ are reflected. On the other hand, when $\boldsymbol{E}_{1} \neq 0$, from curves (2) and (4) in Figure 4(a), a $\mathrm{D}^{+}$ion in the range of $0^{\circ} \geq \theta_{a} \gtrsim-3^{\circ}$ are reflected, but a $\mathrm{D}^{+}$ion in the range of $-3^{\circ} \gtrsim \theta_{a} \gtrsim-36^{\circ}$ is not reflected, that is, the acceleration by $\boldsymbol{E}_{1}$ has brought about such a disadvantage for reflection of a $\mathrm{D}^{+}$ ion. We missed previously this factor. About relativistic electrons, such a problem does not arise, because the second term increasing together with $v_{o z}^{2}$ within the root of (9) is much more predominant than the first term within the root in magnitude. In Figure 4(a), Figure 4(b), from comparison between curves (2) and (3), relativistic effects are seen from around $\theta_{a} \simeq-50^{\circ}$. In the case where $\ell$ $=110 \mathrm{~m}$ (Figure $4(\mathrm{~b})$ ), it is seen that a $\mathrm{D}^{+}$ion starting from plane (a) in the range of $0^{\circ} \geq \theta_{a} \geq-80^{\circ}$ are reflected all. Under the assumption that every $\mathrm{D}^{+}$ion has the velocity-magnitude of $\bar{v}_{i}$, the loss angle of mirror (a) - (d) becomes nearly zero in the case of $\ell=110 \mathrm{~m}$. But in the case of $\ell=52 \mathrm{~m}$, it is presumed that the loss angle of mirror (a) - (d) will be larger than $3^{\circ}$, due to the minus factor mentioned above with respect to a $\mathrm{D}^{+}$ion escaping from plane (a) with a velocity-component in the direction of $-z$ near the $y$ - $z$ plane.

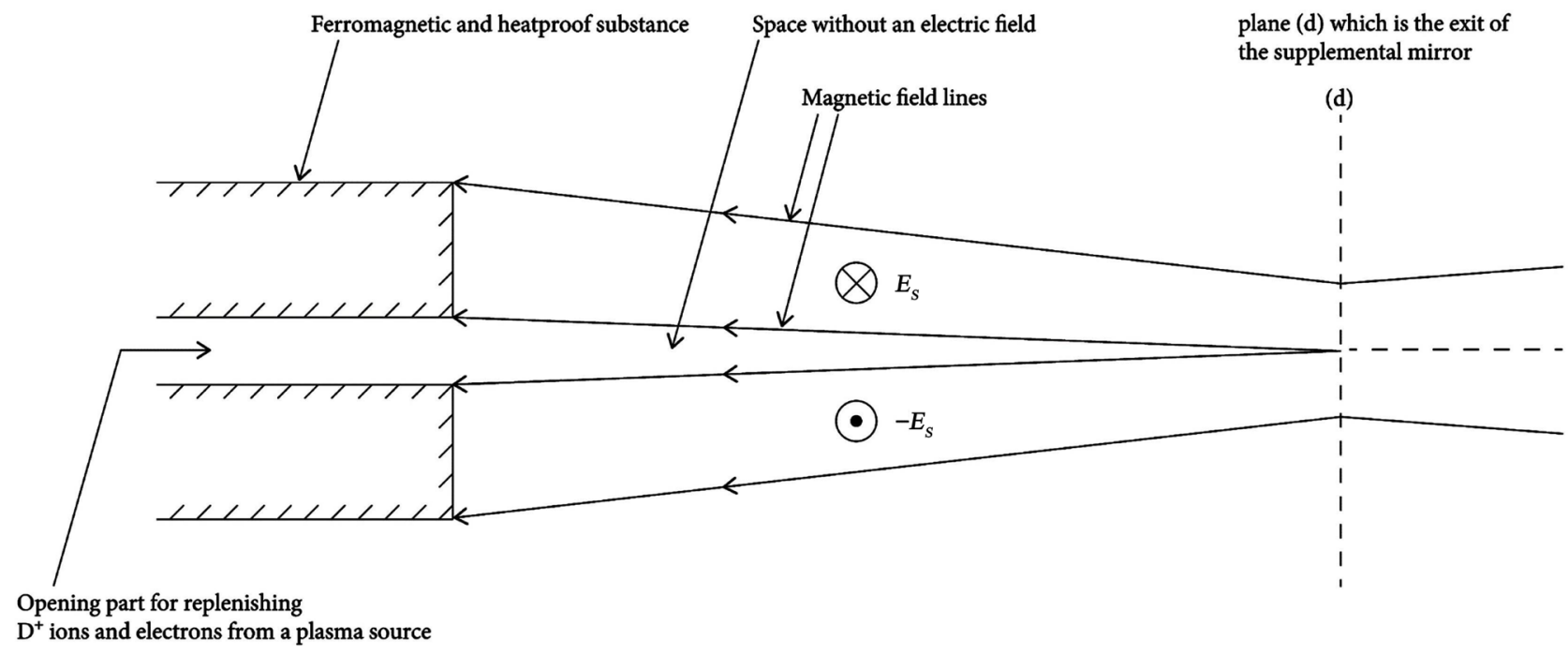

Figure 5. A schematic diagram of an apparatus for replenishing a large quantity of $\mathrm{D}^{+}$ions and electrons. Static electric fields $\pm \boldsymbol{E}_{S}$ are ones for decreasing the number of charged particles colliding with the metal plate by the forces of $\pm \boldsymbol{E}_{S} \times \boldsymbol{B}$. Escaping $\mathrm{D}^{+}$ions must be sent to a plasma source. 


\section{Conclusion}

We have made it clear that, in order to reclaim escaping $\mathrm{D}^{+}$ions whose incident directions make small angles for a direction of $\boldsymbol{B}$ by a magnetic mirror of a linear type, a very long cyclotron resonance space is necessary. Though the loss angle, about $5^{\circ}$, of the mirror (a) - (d) designed in Section 3 is presumed to be still too large from the viewpoint of plasma confinement, the mirror (a) - (d) shown in Figure 1 is a sufficiently too long apparatus. Therefore, we consider that, for shortening a length of acceleration space, a powerful means replenishing a large quantity of $\mathrm{D}^{+}$ions and electrons from the outside ought to be introduced. We show with Figure 5 an idea about a means for replenishment. This apparatus must be protected from heating and damage of the metal surface due to collisions of escaping $\mathrm{D}^{+}$ions.

\section{Conflicts of Interest}

The authors declare no conflicts of interest regarding the publication of this paper.

\section{References}

[1] Nagata, M. and Sawada, K. (2018) EPJ Techniques and Instrumentation, 5, 1.

[2] Turlapov, A.V. and Semenov, V.E. (1998) Physical Review E, 57, 5.

[3] Caspi, R. and Jerby, E. (1999) Physical Review E, 60, 2.

[4] Savrukhin, P.V. and Shestakov, E.A. (2016) Physics of Plasmas, 23, 112509-1.

[5] Soldatkina, E., Anikeev, M., Bagryansky, P., Korzhavina, M., Maximov, V., Savkin, V., Yakovlev, D., Yushmanov, P. and Dunaevsky, A. (2017) Physics of Plasmas, 24, 022505-1. 